\title{
Article
}

\section{Kinematics of the Ship's Wake in the Presence of a Shear Flow}

\author{
Igor Shugan ${ }^{1,2, *}$ and Yang-Yih Chen ${ }^{1}$ \\ 1 Department of Marine Environment and Engineering, National Sun Yat-Sen University, \\ Kaoshiung 804, Taiwan; yichen@mail.nsysu.edu.tw \\ 2 Laboratory of Shelf and Sea Coasts, Shirshov Institute of Oceanology, Russian Academy of Sciences, \\ 117997 Moscow, Russia \\ * Correspondence: ishugan@rambler.ru
}

check for updates

Citation: Shugan, I.; Chen, Y.-Y. Kinematics of the Ship's Wake in the Presence of a Shear Flow. J. Mar. Sci. Eng. 2021, 9, 7. https://dx.doi.org/10.3390/ jmse9010007

Received: 15 November 2020 Accepted: 21 December 2020 Published: 23 December 2020

Publisher's Note: MDPI stays neutral with regard to jurisdictional claims in published maps and institutional affiliations.

Copyright: () 2020 by the authors. Licensee MDPI, Basel, Switzerland. This article is an open access article distributed under the terms and conditions of the Creative Commons Attribution (CC BY) license (https: / / creativecommons.org/ licenses/by/4.0/).

\begin{abstract}
We present the kinematic model of the ship wake in the presence of horizontal subsurface current linearly varying with the depth of water. An extension of the Whitham-Lighthill theory for calm water is developed. It has been established that the structure of ship waves under the action of a shear flow can radically differ from the classical Kelvin ship wake model. Co propagating ship and shear current lead to increasing the total wedge angle up to full one $180^{\circ}$ and decreases for the counter shear current. At relatively large unidirectional values of the shear current, cusp waves in the vicinity of the wedge boundary are represented by transverse waves and, conversely, by diverging waves directed almost perpendicular to the ship track for the opposite shear current. The presence of a shear flow crossing the direction of the ship's movement gives a strong asymmetry of the wake. An increase in the perpendicular shear flow leads to an increase in the difference between the angles of the wake arms. The limiting value of the shear current corresponds to one or both arms angles equal to $90^{\circ}$. Transverse and divergent edge waves for this limiting case coincide.
\end{abstract}

Keywords: ship wake; wake angle; transverse and divergent waves; shear current

\section{Introduction}

The structure of waves on the water surface behind a ship, called a wake, was first examined by Lord Kelvin (Thomson) in 1887 [1]. Starting at approximately several lengths of the ship behind it, the wake geometry and wave pattern are quite universal and independent of the ship's speed and shape in deep water conditions. The wake consists of two sorts of waves: transverse waves with crests across the ship's path and longitudinal waves, moving outward. They are placed in a wedge region behind the ship, and the angle of the wake is approximately $39^{\circ}$. The wake's edges with the maximum amplitude of the longitudinal waves are called Kelvin arms, and the waves in this area are called the cusp waves. The Kelvin solution's main features were reproduced by Lighthill and Whitham [2,3], who suggested the kinematics of the ship wave's model.

Comparison of these theoretical results with observations revealed that, although there is generally acceptable qualitative agreement, there are essential differences between the predicted and measured geometry of the Kelvin diagram in the ship's wake. The main difference in the ship's wake structure is the significant variability of the wake angles [4-6]. In the wake, the half Kelvin angle can be as narrow as $9^{\circ}$ or much wider, up to full $90^{\circ}[6,7]$.

The wake angle statistics observed by the synthetic aperture radar (SAR) method [7] show that, in general, wake angles are narrower than the Kelvin results. Another feature is the asymmetry of the ship's wake's observed shape-very often, only one of the Kelvin arms is visible on SAR images [7-10].

SAR techniques used to detect ships have greatly contributed to the study of ship tracks over the past few decades. Ships may not even be visible on radar images due to their small size, but the ship's trail extends over kilometers and can provide essential information about the ship, its length, speed, direction of movement, etc. [11,12]. Speed estimation from 
radio images helps to track vessels, which is an important part of shipping management. In recent years, several methods for recognizing radar images have been proposed to analyze ship tracks. The direction of movement of the vessel and its speed can be estimated both by the wake's angle and by the length of waves in the cusp region of the wake [12-15]. Thus, a quantitative assessment of the geometry of Kelvin arms is one of the practically demanded tasks. Much of the ship's energy is lost within the ship's wake [16-18], so the properties of ship waves and wave resistance are particularly important in shipbuilding.

The difference between the Kelvin scheme and the ship track observations can be attributed to several reasons arising from the model's basic assumptions. Modeling a ship's wake in calm water and in deep water approaching is one of the main reasons for this discrepancy. Wind waves and a finite sea depth approach can significantly alter a ship's wake $[19,20]$. The wake angle and the cusp waves' characteristics in this case strongly depend on the ship's speed and the depth of the water. The use of the theory of linear waves on water significantly simplified the task [21,22] and, in addition to nonlinearity, did not consider all the effects of the hull shape, the interaction of bow and stern waves, which does not allow adequately simulating the flow field near the ship. Modern ideas about the structure of the ship's wake are presented in Figure 1 [19].

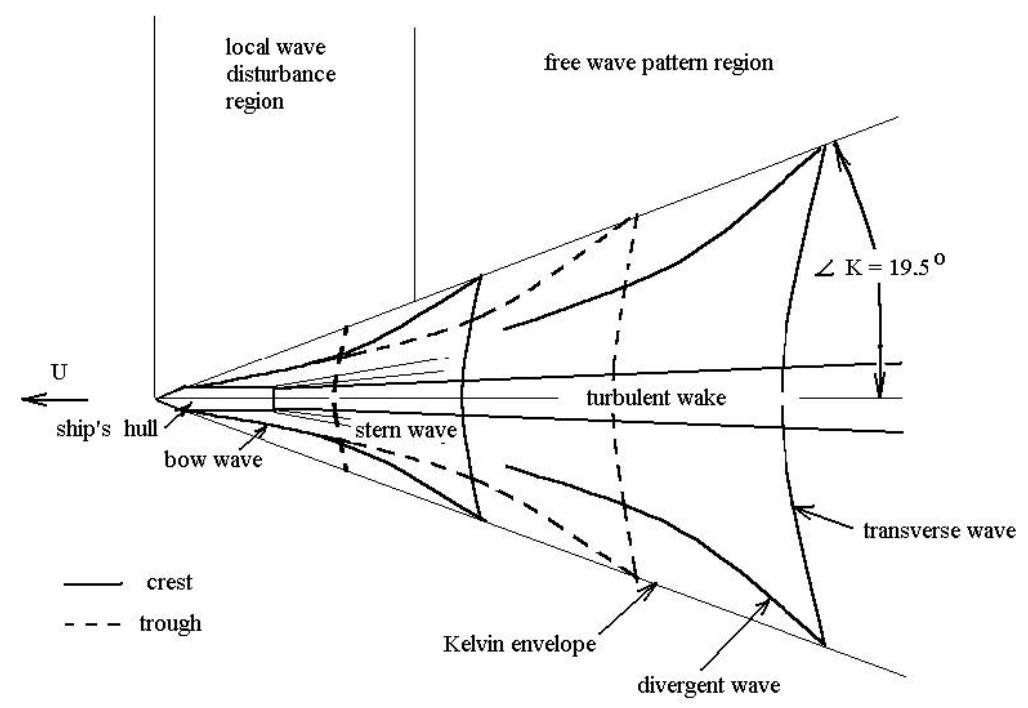

Figure 1. Structure of the ship wake.

The ship waves' structure in the presence of horizontal shear current that changes with water depth can be strongly different from the classical one and exhibit some conceptually new properties. Depth sheared currents are often observed in coastal waters due to wind produced drift, tides, and underwater currents [23,24].

A long list of works is devoted to propagating surface waves in the presence of a horizontal flow that changes with the depth of the fluid. Most of them consider twodimensional interaction in the approximation of constant flow vorticity [23-27]. This provides a significant simplification of the problem because the theory of potential waves is applicable in this case. The three-dimensional problem of the interaction of the transverse current with waves was mainly developed in the last decade [28,29]. The dispersion relation for waves indicates a significant difference in the characteristics of waves depending on their directions with respect to the direction of the shear current.

One of the iconic three-dimensional tasks is studying the structure of the ship's wake on a shear flow. A recent study [30] showed a strong dependence of the wake structure on the shear flow characteristics. The classical Kelvin model, based on consideration of the ship as a moving pressure distribution, was re-analyzed, and the stationary phase method was applied to calculate the far-field wake. It was shown that the angle of the ship's wake could be either large or smaller, and the wake can lose its symmetry due to 
three-dimensional interaction with the shear current. The significant effects of finite water depth were included in consideration of this problem in [17].

Another possibility of analyzing the influence of horizontal shear current on the ship's wake is developing an extended kinematic model of the wake, originally proposed in $[2,3]$. This is the purpose of this study. A model of propagating ship waves in the presence of a constant vorticity flow for an infinite water depth is considered. The advantage of the presented kinematic model is its relative simplicity, which makes it possible to adequately describe the main features of the wake on the shear current. Explicit formulas describe the geometry of the ship's wave structure depending on the magnitude of the shear current's vorticity and its direction relative to the ship's path.

The structure of the paper is as follows. Section 2 presents the basic kinematic model of the ship wake in the presence of horizontal shear current, describes the method of solving, and presents the results. Section 3 describes the results of waves modeling for three variants of the mutual ship path and current directions:

- Collinear (Section 3.1),

- $\quad$ cross (Section 3.2),

- $\quad$ inclined (Section 3.3).

Conclusions are made in Section 4.

\section{Kinematic Model of the Ship Wake}

Let us consider plane surface waves with a vector of wavenumber $\vec{k}=\left(k_{X}, k_{Y}\right)=$ $(-k \operatorname{Cos} \psi, k \operatorname{Sin} \psi)$ (see Figure 2), forming the ship's wake, in the presence of a horizontal shear flow $\vec{U}_{c}=\vec{U}_{0}+\vec{U}_{0}^{\prime} z$, linearly varying with the water depth $z$. The moving frame of reference has the ship fixed at its origin $(\mathrm{P})$ with the uniform surface current $\vec{U}=-\left(\vec{U}_{\text {ship }}+\vec{U}_{0}\right)$ moving in the positive direction along the X-axis. $\vec{U}_{\text {ship }}$ is the vector of the ship's speed relative to the water surface, $\vec{U}_{0}^{\prime}=\left(\vec{U}_{0 X}^{\prime}, \vec{U}^{\prime}{ }_{0 Y}\right)$ is the constant horizontal vector of a shear flow gradient. Our study aims to analyze the structure of stationary ship waves in the presence of a given shear current $\vec{U}_{c}$.

The kinematics model for a ship wave's wake in calm water first was suggested by Lighthill and Whitham [2,3]. We extend the theory for the wave's propagation in the presence of the shear current with constant vertical shear $\vec{U}_{0}^{\prime}$. The deepwater approximation $k h \gg 1$ is assumed, which is acceptable for the ship wave's pattern with the length of the ship and waves much less than the depth of the fluid $h$. We will also take the approximation of an incompressible and inviscid fluid.

The linear dispersion equation for surface gravity waves in the problem under consideration includes the Doppler frequency shift and the effect of the shear flow and has the well-known form [29,30]:

$$
\mathrm{G}\left(k_{X}, k_{Y}\right)=(\omega-\vec{k} \cdot \vec{U})^{2}+(\omega-\vec{k} \cdot \vec{u}) \frac{\left(\vec{k} \cdot \vec{U}_{0}^{\prime}\right)}{|\vec{k}|}-|\vec{k}| g=0
$$

where $\omega$ is the frequency of waves, $g$ is gravity acceleration. 


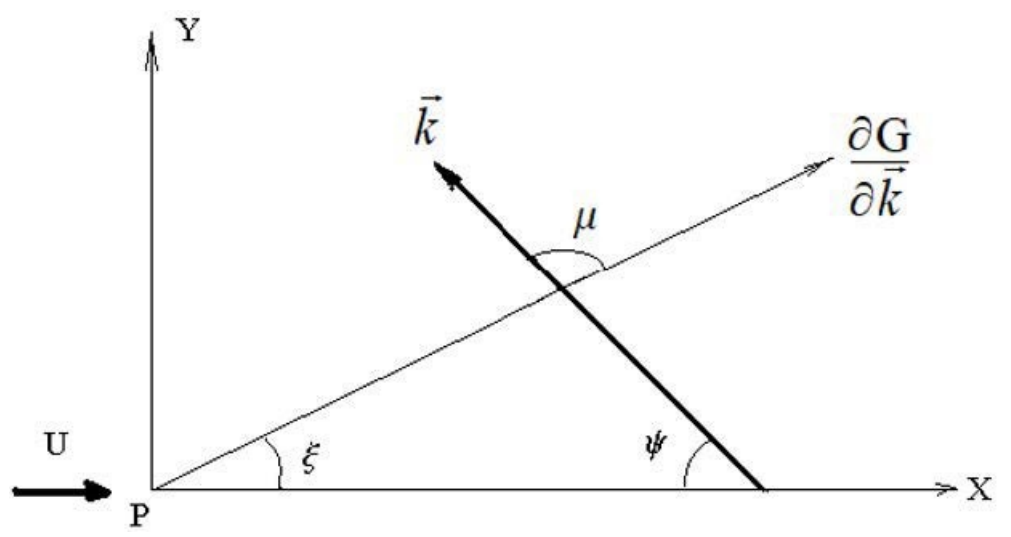

Figure 2. The geometry of waves behind the ship. $\vec{U}$ is the flow velocity, $\vec{k}$-wave number vector of surface waves, $(\xi, \psi)$-ray and wave number vector angles, respectively; vector $\frac{\partial G}{\partial \vec{k}}=\left(G_{k_{X}}, G_{k_{Y}}\right)$ expresses the ray direction $\tan \xi=G_{k_{Y}}\left(k_{X}, k_{Y}\right) / G_{k_{X}}\left(k_{X}, k_{Y}\right)$.

The kinematics of wave motion is described by the law of conservation of the number of waves [31]:

$$
\frac{\partial \vec{k}}{\partial T}+\nabla \omega=0
$$

and consistency equation for the wavenumber function:

$$
\frac{\partial k_{X}}{\partial Y}=\frac{\partial k_{Y}}{\partial X}
$$

We consider a stationary problem with zero frequency of the field of surface waves in the selected coordinate system; therefore, Equation (2) is identically valid. In polar coordinates, we have (Figure 1): $k_{X}=-\operatorname{Cos}(\psi)|\vec{k}|, k_{Y}=\operatorname{Sin}(\psi)|\vec{k}|,-\pi / 2<\psi<\pi / 2$.

Finally, we have the pair of Equations (1) and (3) for two unknown functions $\left(k_{X}, k_{Y}\right)$. The dependence $k_{X}=f\left(k_{Y}\right)$ can be found from the dispersion relation (1), and the quasilinear Equation (3) can be solved by the method of characteristics.

Equation (3) for $k_{Y}$ function may be rewritten in the following form:

$$
\frac{\partial k_{Y}}{\partial X}=f^{\prime}\left(k_{Y}\right) \frac{\partial k_{Y}}{\partial Y}
$$

The system of characteristics for this equation is:

$$
\frac{d Y}{d X}=-f^{\prime}\left(k_{Y}\right), \frac{d k_{Y}}{d X}=0
$$

For the point source $\mathrm{P}$, we have the central wave and characteristics—straight rays with a constant wavenumber along the direction of ray $\xi$ :

$$
\tan \xi=Y / X=-f^{\prime}\left(k_{Y}\right)=\frac{G_{k_{Y}}\left(k_{X}, k_{Y}\right)}{G_{k_{X}}\left(k_{X}, k_{Y}\right)} ; k_{Y}, k_{X}=\text { const }
$$

where $\xi$ is the ray angle (see Figure 1).

A complete picture of the wave pattern behind the ship can also be drawn with lines with the same wave phase and with crest lines. The expression for the phase function may be found from its definition:

$$
\theta(X, Y)=\int_{0}^{\vec{X}} \vec{k} \cdot d \vec{X}
$$


When calculating the integral, you can use any trajectory, since the wave number field is irrotational. The rays $\xi=$ const are convenient for calculation, since the wave number $\vec{k}$ along them is constant, and we have:

$$
\theta(X, Y)=r|\vec{k}| \operatorname{Cos}(\mu), \mu=\pi-\psi-\xi,
$$

where $r$-is the distance from the origin, $\mu$-is the angle between the vectors of the ray and the wavenumber (see Figure 1).

\section{Results}

\subsection{Collinear Propagation of the Ship and Shear Flow $\left(U_{0 X^{\prime}} \neq 0, U_{0 Y^{\prime}}=0\right)$}

We start with the simplest case of collinear ship propagation and shear flow along the $\mathrm{X}$-axis. For steady-state waves in the chosen moving frame of reference with zero frequency $\omega=0$, the dispersion relation (1) takes the form:

$$
\mathrm{G}\left(k_{x}, k_{y}\right)=k_{X}^{2}\left(\frac{U^{2}}{g}+\frac{W_{X}}{|\vec{k}|}\right)-|\vec{k}|=0, W_{X}=-\frac{U U_{0 X}{ }^{\prime}}{g},
$$

where $U$ is the speed of surface flow, $W_{X}$ is a dimensionless parameter that characterizes the vorticity of the underlying flow. The ray Equation (5) takes the form:

$$
\tan \xi=Y / X=\frac{G_{k_{Y}}\left(k_{X}, k_{Y}\right)}{G_{k_{X}}\left(k_{X}, k_{Y}\right)}=\frac{W_{X} \frac{\tan \psi}{1+\tan ^{2} \psi}+\tan \psi}{-W_{X} \frac{1}{1+\tan ^{2} \psi}+1+2 \tan ^{2} \psi}
$$

The directions of ship waves $\psi:-k_{Y} / k_{X}=\tan (\psi)$ depending on the ray angle $\tan \xi$ are shown in Figure 3 for unidirectional with the ship $\left(W_{X}>0\right)$ and oncoming $\left(W_{X}<0\right)$ shear current.

Because $k_{X}=-|\vec{k}| \operatorname{Cos}(\psi)<0$, only the range $-\pi / 2<\psi<\pi / 2$ is permissible. The dependence (8) is evidently symmetrical concerning the $X$-axes, so we present only the plane's upper half $0<\psi<\pi / 2$.

Two types of waves are represented within the ship's wake: transverse waves (crests intersect the ship's trajectory) and divergent waves (crests are approximately parallel to the ship's trajectory and move outward), which is consistent with Kelvin's results [1].
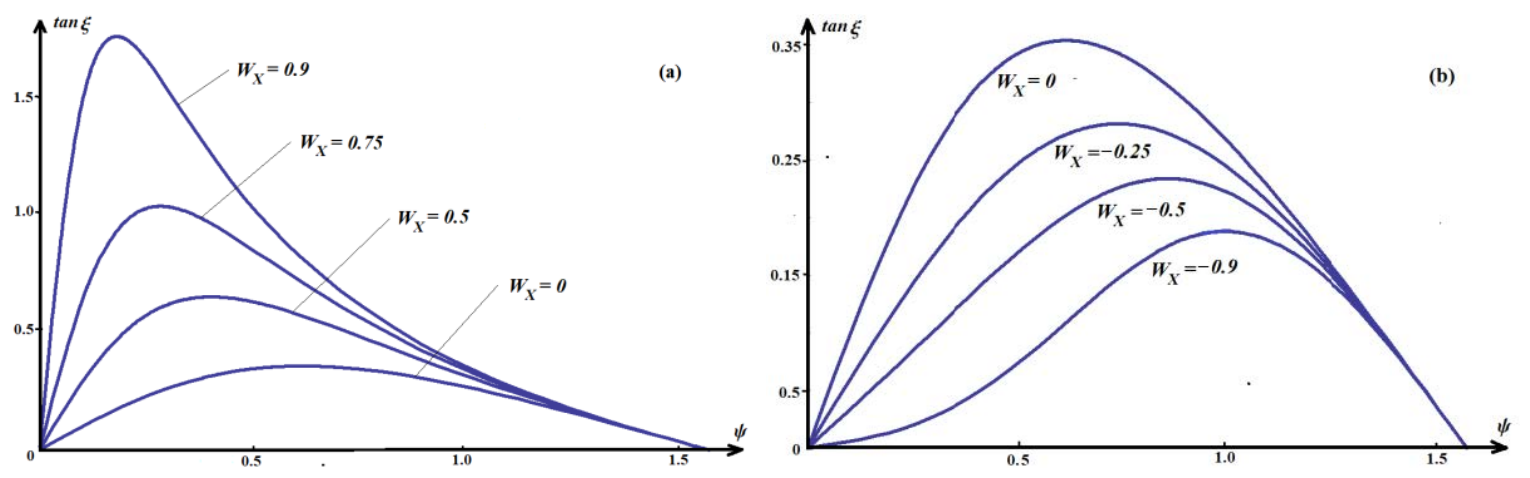

Figure 3. Directions of ship waves $\psi:-k_{Y} / k_{X}=\tan (\psi)$ depending on the ray angle $\tan \xi$ for different values of shear current: (a) Unidirectional with the ship $\left(W_{X}>0\right)$ shear current; (b) Oncoming $\left(W_{X}<0\right)$ shear current.

The ship's wake structure drastically depends on the magnitude of the shear current. The ship wake's total wedge angle increases for a unidirectional with ship shear current 
up to $180^{\circ}$ for $W_{X}=1$ (Figure 3a) and decreases for the counter shear current (Figure 3b). At relatively large positive values of the shear current, cusp ship waves with the angle $\psi_{m}$ in the wedge boundary's vicinity are mainly represented by transverse waves and, conversely, by divergent waves for the counter shear current. The cusp wavelength $\lambda_{m}=\frac{2 \pi U^{2}}{g}\left(\frac{1}{\cos ^{2} \psi_{m}}-W_{X}\right)^{-1}$ increases with increasing unidirectional shear current up to infinity.

The lines of the constant phase $\theta(X, Y)=$ const in polar coordinates $(r, \xi)$ may be presented in the parametric form in terms of $\psi$ :

$$
\begin{aligned}
& r=-\frac{\theta U^{2}}{g} \frac{\sqrt{1+\tan ^{2} \mu}}{\left(1+\tan ^{2} \psi-W_{X}\right)} ; \tan \mu=\frac{2\left(1+\tan ^{2} \psi\right) \tan \psi}{W_{X}-\left(1+\tan ^{2} \psi\right)} ; \\
& \tan \xi=Y / X=\frac{W_{X} \frac{\tan \psi}{1+\tan ^{2} \psi}+\tan \psi}{-W_{X} \frac{1}{1+\tan ^{2} \psi}+1+2 \tan ^{2} \psi} .
\end{aligned}
$$

The value of $\theta$ is negative. Phase lines can also be represented in Cartesian coordinates:

$$
\begin{aligned}
& X=-\frac{\theta U^{2}}{g} \frac{\sqrt{\left(1-\frac{W_{X}}{1+\tan ^{2} \psi}\right)^{2}+4 \tan ^{2} \psi}}{\left(1-\frac{W_{X}}{1+\tan ^{2} \psi}\right)^{2}} \frac{\cos ^{2} \psi}{\sqrt{1+\tan ^{2} \xi}} ; \\
& Y=-\frac{\theta U^{2}}{g} \frac{W_{X} \frac{\tan \psi}{1+\tan ^{2} \psi}+\tan \psi}{-W_{X} \frac{1}{1+\tan ^{2} \psi}+1+2 \tan ^{2} \psi} \frac{\sqrt{\left(1-\frac{W_{X}}{1+\tan ^{2} \psi}\right)^{2}+4 \tan ^{2} \psi}}{\left(1-\frac{W_{X}}{1+\tan ^{2} \psi}\right)^{2}} \frac{\cos ^{2} \psi}{\sqrt{1+\tan ^{2} \xi}} .
\end{aligned}
$$

Typical examples of wave patterns are shown in Figure 4 for unidirectional $\left(W_{X}=0.75\right)$ and opposite $\left(W_{X}=-0.75\right)$ shear current.
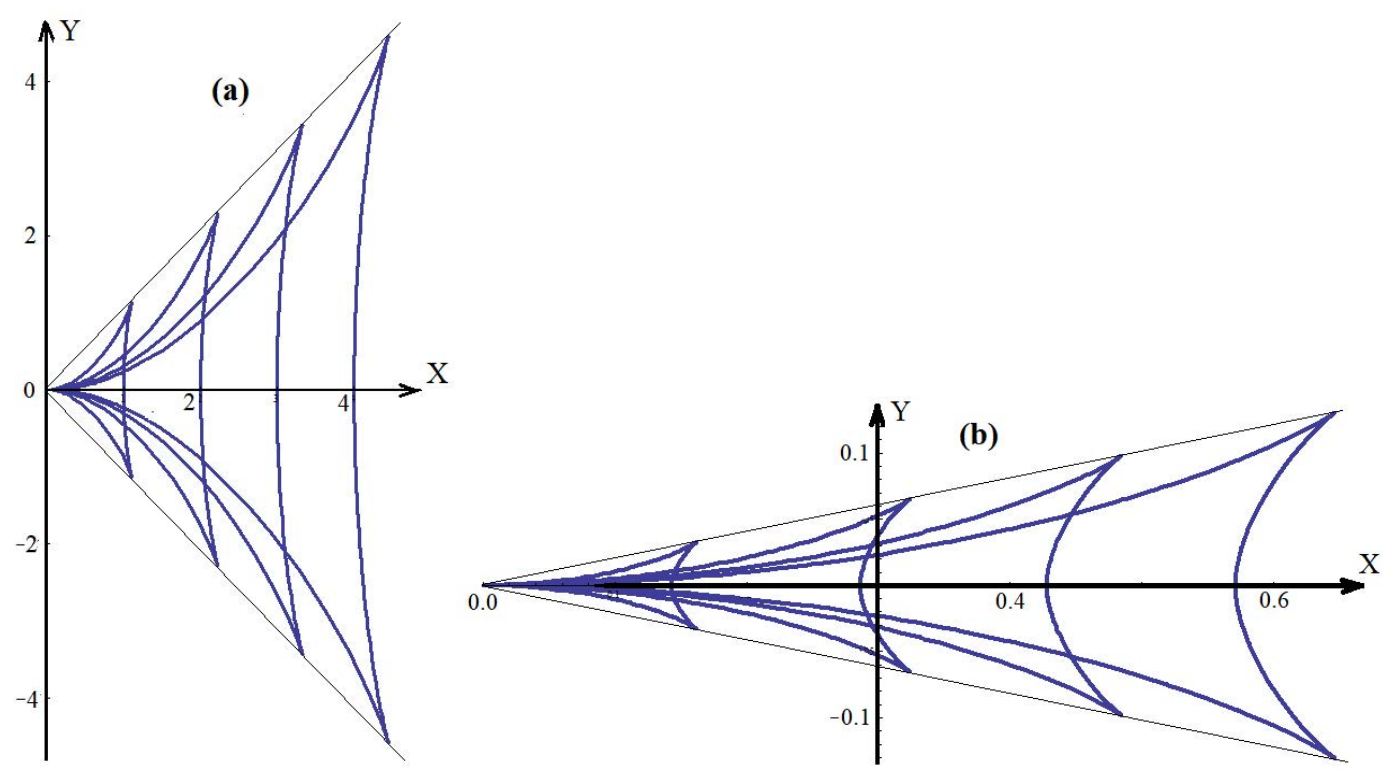

Figure 4. The lines of the constant phase $\theta(X, Y)=$ const for different values of shear current: (a) Unidirectional with the ship $\left(W_{X}=0.75\right)$ shear current; (b) Opposite $\left(W_{X}=-0.75\right)$ shear current.

The phase lines are symmetrical about the $\mathrm{X}$-axis. The wavenumber vectors at each point are perpendicular to the phase line. All phase lines have a cusp that separates transverse and divergent waves. The phase lines' geometry is quite similar to that pre- 
sented in [30], which can serve as a test and clearly demonstrates the applicability of the presented model.

\subsection{Cross Direction of the Shear Flow}

Next, we analyze the effect of the shear current perpendicular to the ship's speed $\left(W_{Y} \neq 0, W_{X}=0\right)$. For steady-state waves in the chosen moving frame of reference with zero frequency $\omega=0$, the dispersion relation (1) has the form:

$$
\mathrm{G}\left(k_{x}, k_{y}\right)=k_{X}^{2} \frac{U^{2}}{g}+k_{X} k_{Y} \frac{W_{Y}}{|\vec{k}|}-|\vec{k}|=0, W_{Y}=-\frac{U U_{0 Y^{\prime}}}{g} .
$$

The ray Equation (5) is the following:

$$
\tan \xi=Y / X=\frac{\frac{W_{Y}}{1+\tan ^{2} \psi}+\tan \psi}{W_{Y} \tan \psi \frac{1+2 \tan ^{2} \psi}{1+\tan ^{2} \psi}+1+2 \tan ^{2} \psi}
$$

Equation (12) is invariant under the transformation: $W_{Y} \rightarrow-W_{Y}, \psi \rightarrow-\psi, \xi \rightarrow-\xi$, therefore, we consider only positive values of $W_{Y}>0$. The directions of ship waves $-k_{Y} / k_{X}=\tan (\psi)$ depending on the ray angle $\xi$ are shown in Figure 5a. Dependence (12) is clearly asymmetric about the $\mathrm{X}$-axis; therefore, we presented the entire variation range $-\pi / 2<\psi<\pi / 2$. A typical sketch of the phase lines for the shear value $\left(W_{Y}=0.75\right)$ is shown in Figure $5 b$.
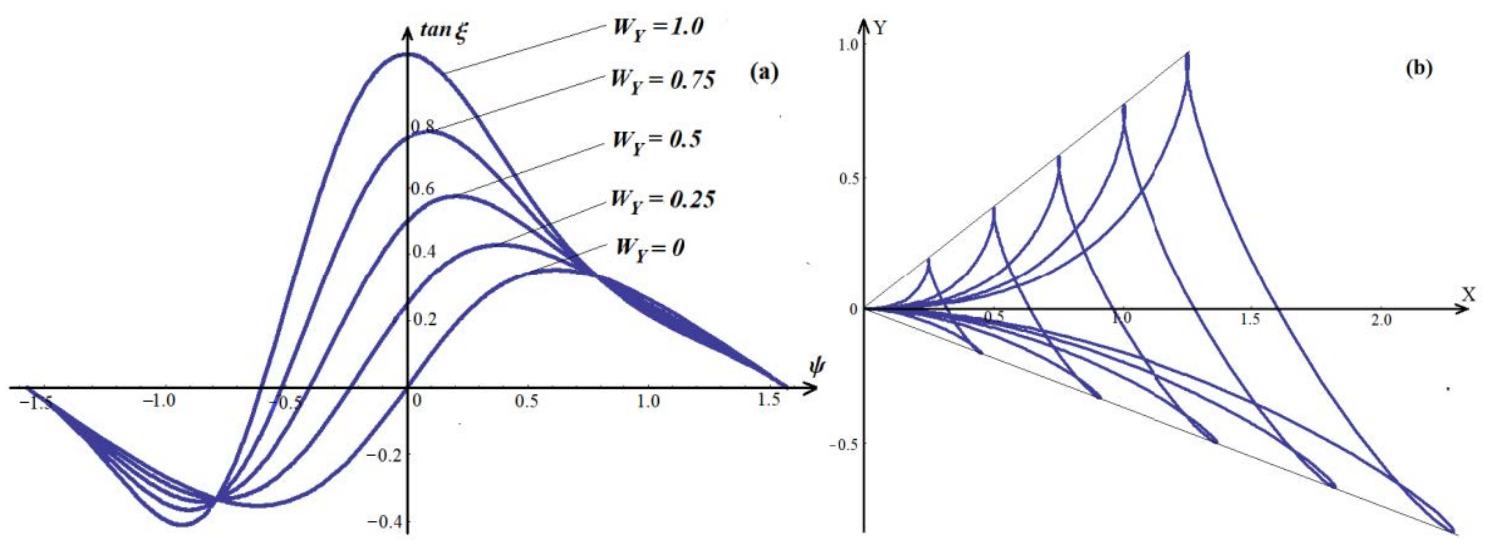

Figure 5. Cross interaction of waves and shear current: (a) Directions of ship waves $-k_{Y} / k_{X}=\tan (\psi)$ depending on the ray angle $\xi$ for different values $W_{Y}>0 ;(\mathbf{b})$ Phase lines $\theta(X, Y)=$ const for the current shear value $\left(W_{Y}=0.75\right)$.

Transverse waves have a negative direction $(\psi<0, \xi=0)$ along the trajectory of the ship. Two wake arms have different absolute values of angles (see Figure 5a,b). The positive wake arm angle $\xi_{m}$ increases with the flow vorticity $W_{Y}$ and reaches the value $45^{\circ}$ for $W_{Y}=1$, while the () value limits the negative arm angle. Figure $5 \mathrm{~b}$ of the constant phase lines demonstrates the strong asymmetry of the ship wake in the presence of the cross-shear flow.

Cusp waves are placed along the arms of the wake. The angle of inclination of the cusp waves $\psi_{m}$ in depending on $W_{Y}$ is shown in Figure 6. It decreases monotonically with $W_{Y}$ and is equal to zero for $W_{Y}=1$-transverse cusp waves propagate along the direction of the ship motion. A further increase in shear flow leads to the negative direction of the arm angles' cusp waves and values $\xi_{m}>\pi / 4$. The limit value of the shear flow $W_{Y}=2$ corresponds to arms angles $\xi_{m}= \pm 90^{\circ}$ and zeroing the group velocity of the cusp waves. The transverse and divergent edge waves for that limiting case coincide and have an angle 
$\psi_{m}=-45^{\circ}$. The resulting limit value $W_{Y}$ coincides with that given in [30], which further validates our model.

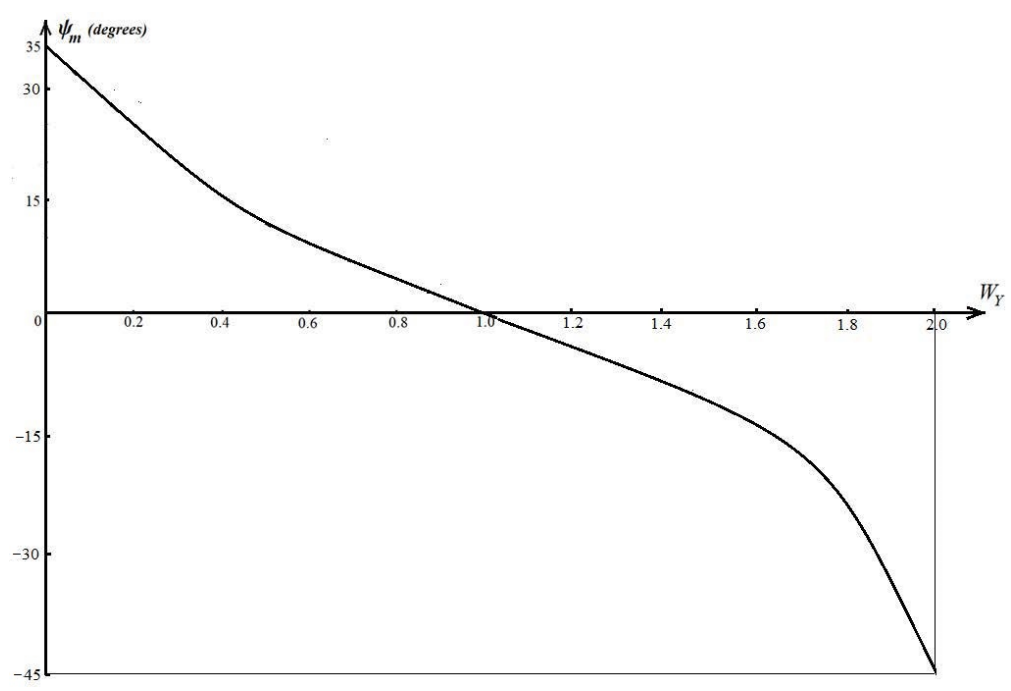

Figure 6. The angle of inclination of the cusp waves $\psi_{m}$ in depending on shear flow $W_{Y}$.

\subsection{Inclined Direction of the Shear Flow}

In the general case, it is necessary to investigate the shear flow's oblique direction $\left(W_{Y} \neq 0, W_{X} \neq 0\right)$.

Dispersion relation (1) has the form:

$$
\mathrm{G}\left(k_{x}, k_{y}\right)=k_{X}^{2} \frac{U^{2}}{g}+k_{X}^{2} \frac{W_{X}}{|\vec{k}|}+k_{X} k_{Y} \frac{W_{Y}}{|\vec{k}|}-|\vec{k}|=0, W_{Y}=-\frac{u U_{0 Y^{\prime}}}{g}, W_{X}=-\frac{u U_{0 Y^{\prime}}}{g} .
$$

The ray direction Equation (5) is:

$$
\tan \xi=Y / X=\frac{W_{X} \frac{\tan \psi}{1+\tan ^{2} \psi}+\frac{W_{Y}}{1+\tan ^{2} \psi}+\tan \psi}{-W_{X} \frac{1}{1+\tan ^{2} \psi}+W_{Y} \tan \psi \frac{1+2 \tan ^{2} \psi}{1+\tan ^{2} \psi}+1+2 \tan ^{2} \psi}
$$

Equation (14) is obviously invariant under the transformation: $W_{Y} \rightarrow-W_{Y}, \psi \rightarrow-\psi$, $\xi \rightarrow-\xi$, therefore, we consider only positive values of $W_{Y}>0$.

Examples of ship waves' directions $-k_{Y} / k_{X}=\tan (\psi)$ depending on the ray angle $\xi$ are shown in Figure 7 for positive and negative $X$-components of shear flow $W_{X}= \pm W_{Y}$. For each ray angle $\xi$, we still have a pair of ship waves: transverse and divergent; the wake wave's pattern is asymmetric about the $X$-axis. For each value of the shear current, there is a cusp on the ray $\xi=\xi_{m}$ with the wave's direction $\psi=\psi_{m}$. For the positive shear components $W_{X}=W_{Y}=1 / \sqrt{2}$ (see curve $(I V)$ in Figure $7 \mathrm{a}$ ), the edge wave's angles are equal to $\psi_{m}=0$, which means that the waves on the cusp $\xi_{m}=67^{\circ}$ will be strictly parallel to the ship's movement. For opposite signs of shear flow components $W_{X}=-W_{Y}=-1 / \sqrt{2}$ (see curve $(I V)$ in Figure $7 \mathrm{~b}$ ), the corresponding cusp angle will be $\xi_{m}=22^{\circ}$. The shear current for this case satisfies the relationship:

$$
W_{X}^{2}+W_{Y}^{2}=1
$$

A sketch of the phase lines for the shear currents $W_{Y}=1 / \sqrt{2}, W_{X}= \pm 1 / \sqrt{2}$ are presented in Figure 8a,b. 

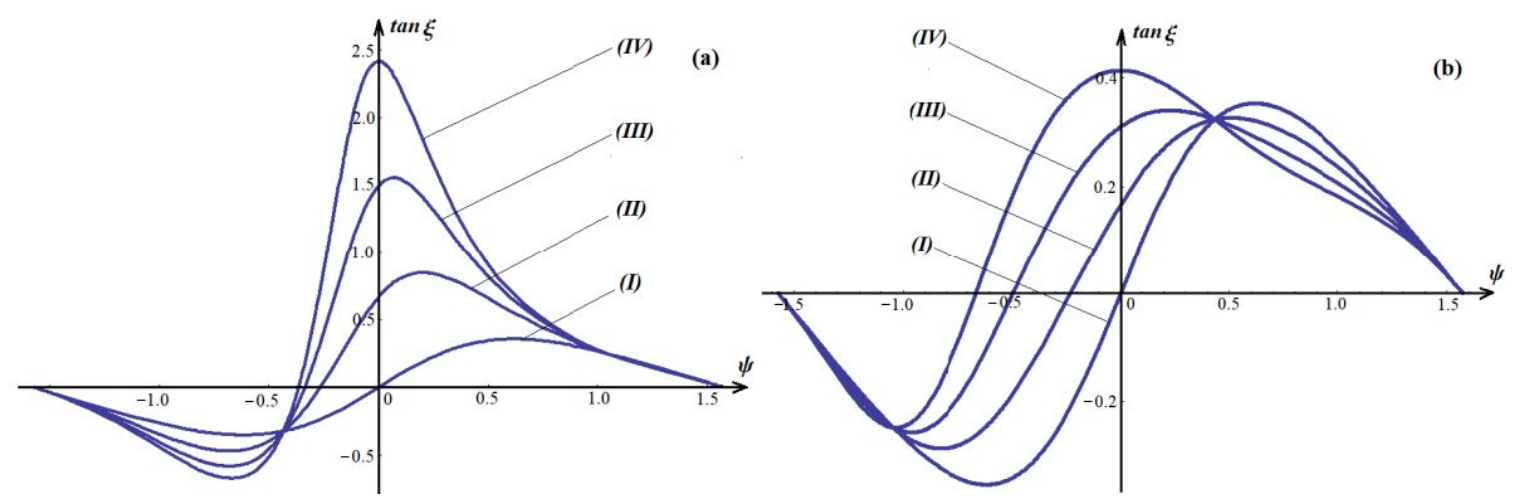

Figure 7. Directions of ship waves $-k_{Y} / k_{X}=\operatorname{tg}(\psi)$ depending on the ray angle $\xi:(\mathbf{a})(I)-\left(W_{X}=W_{Y}=0\right)$, $(I I)-$ $\left(W_{X}=W_{Y}=0.4\right),(I I I)-\left(W_{X}=W_{Y}=0.6\right),(I V)-\left(W_{X}=W_{Y}=1 / \sqrt{2}\right) ;(\mathbf{b})(I)-\left(W_{X}=W_{Y}=0\right),(I I)-\left(W_{X}=-W_{Y}=-0.2\right)$, $(I I I)-\left(W_{X}=-W_{Y}=-0.45\right),(I V)-\left(W_{X}=-W_{Y}=-1 / \sqrt{2}\right)$.
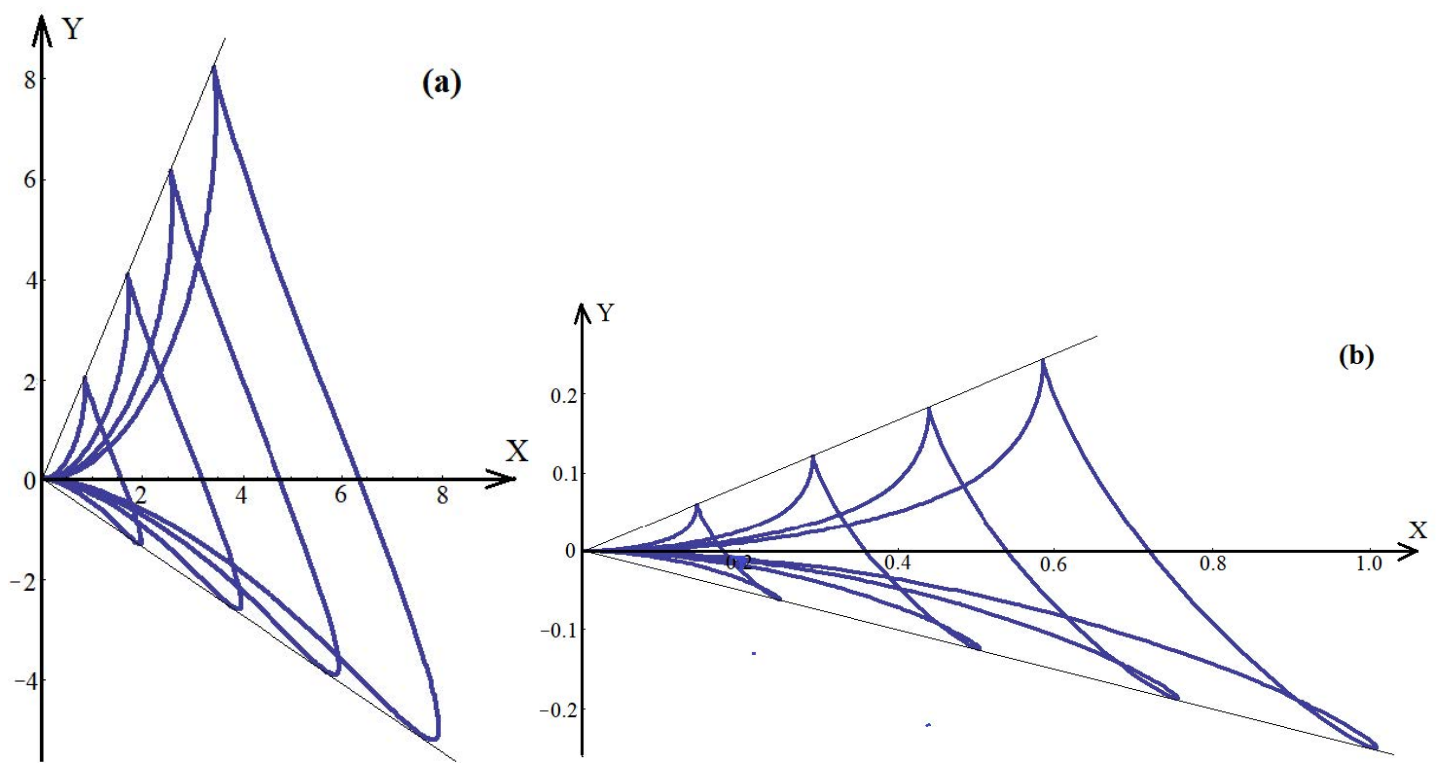

Figure 8. The lines of the constant phase $\theta(X, Y)=$ const for different values of shear current: (a) positive shear components $\left(W_{X}=W_{Y}=1 / \sqrt{2}\right) ;(\mathbf{b})$ Opposite $\left(W_{X}=-W_{Y}=-1 / \sqrt{2}\right)$ shear current components.

A further increase in shear flow leads to a negative direction of cusp waves $\psi_{m}<0$ and arm angles $\xi_{m}>68^{\circ}$. The directions of ship waves $-k_{Y} / k_{X}=\tan (\psi)$ depending on the ray angle $\xi$ are shown in Figure 9 for positive and negative $X$-components of shear flow $W_{X}= \pm W_{Y}, W_{Y}>1 / \sqrt{2}$. A sketch of the phase lines for the shear currents components $W_{Y}=0.82, W_{X}= \pm 0.82$ are presented in Figure 10a,b. The upper limit value of the positive shear flow $W_{X}=W_{Y}=0.92$ corresponds to the arm angle $\xi_{m}=90^{\circ}$ and zeroing the group velocity of the cusp waves $G_{k_{X}}\left(k_{X}, k_{Y}\right)=0$. Transverse and divergent edge waves for this limiting case coincide and have an angle $\psi_{m}=-10^{\circ}$. For the limiting negative shear current component $W_{X}=-W_{Y}=-2.47$, the arms angles are $\xi_{m}= \pm 90^{\circ}$, cusp waves are $\psi_{m}=-44^{\circ}$, and the total wake angle is $180^{\circ}$. 


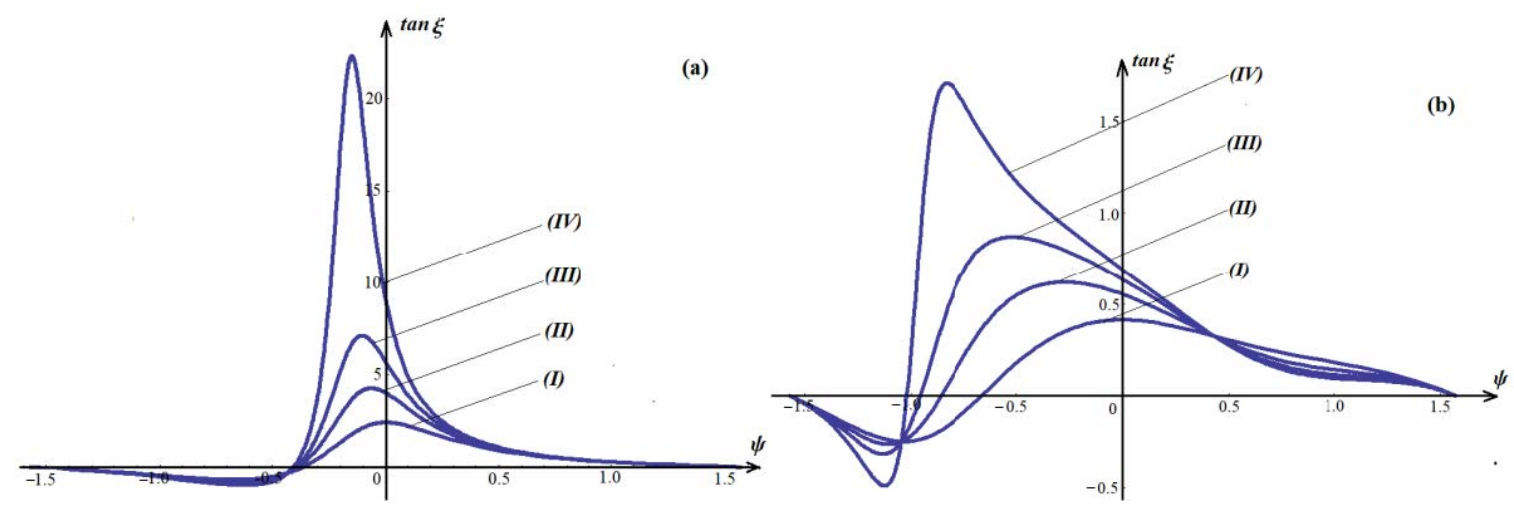

Figure 9. Directions of ship waves $-k_{Y} / k_{X}=\tan (\psi)$ depending on the ray angle $\xi$ : (a) $(I)-\left(W_{X}=W_{Y}=1 / \sqrt{2}\right)$, $(I I)-\left(W_{X}=W_{Y}=0.8\right),(I I I)-\left(W_{X}=W_{Y}=0.85\right),(I V)-\left(W_{X}=W_{Y}=0.9\right) ;(\mathbf{b})(I)-\left(W_{X}=-W_{Y}=-1 / \sqrt{2}\right),(I I)-$ $\left(W_{X}=-W_{Y}=-1.25\right),(I I I)-\left(W_{X}=-W_{Y}=-1.75\right),(I V)-\left(W_{X}=-W_{Y}=-2.25\right)$.
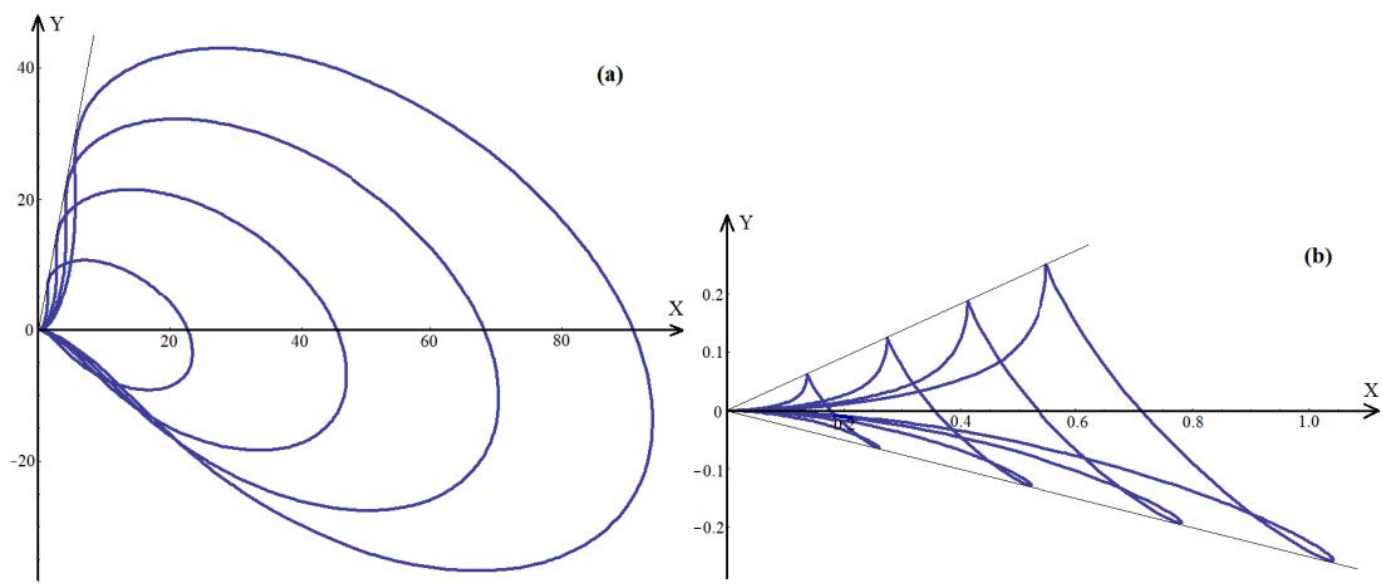

Figure 10. The lines of the constant phase $\theta(X, Y)=$ const: (a) positive shear components $\left(W_{X}=W_{Y}=0.82\right)$; (b) opposite $\left(W_{X}=-W_{Y}=-0.82\right)$ shear current components.

\section{Conclusions}

The structure of ship waves in the presence of a horizontal shear flow, which changes with water depth, can radically differ from the classical Kelvin ship wake model and possess several strikingly new properties. The presented kinematic model of the ship's wake develops the well-known Whitham-Lighthill model and can adequately describe the main features of the stationary picture of the ship's wake.

The ship's collinear propagation and the shear flow result in a symmetrical wake structure concerning the ship's path. There are two types of waves in the wake of the ship: transverse and divergent, which is consistent with the classical results. Co propagating ship and shear current leads to increasing the total wedge angle up to full one $180^{\circ}$ for the no dimensional magnitude of shear $W_{X}=1$ and decreases for the counter shear current. At relatively large unidirectional values of the shear current, cusp waves in the vicinity of the wedge boundary are represented by transverse waves and, conversely, by diverging waves directed almost perpendicular to the ship track for the opposite shear current. The cusp wavelength increases with increasing unidirectional shear current.

The presence of a shear flow crossing the direction of the ship's movement gives a strong asymmetry of the wake. The positive wake arm angle increases with the positive value of shear $W_{Y}$ and reaches the value $45^{\circ}$ for $W_{Y}=1$, while the negative arm angle is limited by the $\left(-22.3^{\circ}\right)$ value. The inclination of the cusp waves decreases monotonically with $W_{Y}$ and is equal to zero for $W_{Y}=1$-transverse cusp waves propagate along the direction of the ship motion. A further increase in the shear flow leads to a negative 
direction of cusp waves and values of the arm angles $\xi_{m}>45^{\circ}$. The limiting value of the shear current $W_{Y}=2$ corresponds to arms angles $\xi_{m}= \pm 90^{\circ}$ and zeroing of the cusp waves' group velocity. Transverse and divergent edge waves for this limiting case coincide and have an angle $\psi_{m}=-45^{\circ}$.

In the general case, the shear flow's oblique direction to the trajectory of the ship also gives the wake asymmetry. The positive wake arm angle increases with the positive values of the shear components, and for $W_{X}^{2}+W_{Y}^{2}=1$ the edge waves will be strictly parallel to the movement of the ship. A further increase in shear flow leads to a negative direction of cusp waves. The upper limit value of the positive shear flow $W_{X}=W_{Y}=0.92$ corresponds to the arm angle $\xi_{m}=90^{\circ}$ and zeroing the group velocity of the cusp waves. Transverse and divergent edge waves for this limiting case coincide and have an angle $\psi_{m}=-10^{\circ}$. For the limiting negative shear current component $W_{X}=-W_{Y}=-2.47$, the arms angles are $\xi_{m}= \pm 90^{\circ}$, cusp waves angles are $\psi_{m}=-44^{\circ}$.

The presented model has a vastly different approach from the classical one, based on consideration of the ship as moving pressure distribution on the sea surface. Asymptotic nontrivial numerical integration for every specific oceanographic situation in the solution's traditional scheme needs much effort and has its restrictions.

Despite its relative simplicity, our kinematic model reproduces some results similar to those obtained according to the classical scheme [30], which, in turn, can serve as a test of the model's applicability. Here we can cite the typical pictures of the phase lines of ship waves, the critical values of the shear flow gradient during cross interaction, etc. As an advantage of the presented model, it can be noted that its analytical solution makes it possible to explicitly describe the structure of ship waves in the entire range of changes in the defining parameters of the problem, and not only its typical behavior and limiting cases.

Author Contributions: Conceptualization, I.S. and Y.-Y.C.; methodology I.S. and Y.-Y.C.; software, I.S.; validation, I.S.; formal analysis, I.S.; investigation, I.S.; resources, I.S. and Y.-Y.C.; writingoriginal draft preparation, I.S.; writing-review and editing I.S. and Y.-Y.C.; visualization, I.S.; supervision, Y.-Y.C.; project administration, Y.-Y.C.; funding acquisition, Y.-Y.C. All authors have read and agreed to the publication of the manuscript.

Funding: The reported study was funding by RFBR and TUBITAK according to the research project 20-55-46005. The study was also funded by the Ministry of Science and Higher Education of the Russian Federation, theme no. 0149-2019-0005.

Acknowledgments: The authors acknowledge the financial support from the Ministry of Science and Technology of Taiwan, under Grant Number MOST 106-2221-E-110-036-MY3 for this study.

Conflicts of Interest: The authors declare no conflict of interest. The funders had no role in the study's design; in the collection, analyses, or interpretation of data; in the writing of the manuscript, or in the decision to publish the results.

\section{References}

1. Thomson, S.W. On ship waves. Proc. Inst. Mech. Eng. 1887, 38, 409-434. [CrossRef]

2. Lighthill, M.J.; Whitham, G.B. On Kinematic Waves: I. Flood Movement in Long Rivers; II. Theory of Traffic Flow on Long Crowded Roads. Proc. R. Soc. Lond. A 1955, 229, 281-345.

3. Whitham, G.B. Linear and Nonlinear Waves; Wiley: New York, NY, USA, 1999.

4. Reed, A.; Milgram, J. Ship Wakes and Their Radar Images. Annu. Rev. Fluid Mech. 2002, 34, 469-502. [CrossRef]

5. Shemdin, O.H. Synthetic Aperture Radar Imaging of Ship Wakes in the Gulf of Alaska. J. Geophys. Res. Ocean. 1990, 95, 16319-16338. [CrossRef]

6. Hennings, I.; Romeiser, R.; Alpers, W.; Viola, A. Radar Imaging of Kelvin Arms of Ship Wakes. Int. J. Remote Sens. 1999, 20, 2519-2543. [CrossRef]

7. Melsheimer, C.; Lim, H.; Shen, C. Observation and Analysis of Ship Wakes in ERS-SAR and SPOT Images. In Proceedings of the 20th Asian Conference on Remote Sensing, Hong Kong, China, 22-25 November 1999; pp. 554-559.

8. Alpers, W.; Romeiser, R.; Hennings, I. On the Radar Imaging Mechanism of Kelvin Arms of Ship Wakes. In Proceedings of the IGARSS'98, Piscataway, NJ, USA, 6-10 July 1998; pp. 1932-1934.

9. Tunaley, J.K.E.; Buller, E.H.; Wu, K.H.; Rey, M.T. The Simulation of the SAR Images of a Ship Wake. IEEE Trans. Geosci. Remote Sens. 1991, 29, 149-155. [CrossRef] 
10. Tunaley, J. The Unsteady Wake from a Body Moving Near an Internal Layer. In Proceedings of the 5th Canadian Conference on Marine Hydromechanics and Structures, St. Johns, NL, Canada, 1-5 July 1999; pp. 5-13.

11. Eldhuset, K. An Automatic Ship and Ship Wake Detection System for Spaceborne SAR images in Coastal Regions. IEEE Trans. Geosci. Remote Sens. 1996, 34, 1010-1019. [CrossRef]

12. Kuo, J.M.; Chen, K.S. The Application of Wavelets Correlator for Ship Wake Detection in SAR Images. IEEE Trans. Geosci. Remote Sens. 2003, 41, 1506-1511.

13. Karakuş, O.; Rizaev, I.; Achim, A. Ship Wake Detection in SAR Images via Sparse Regularization. IEEE Trans. Geosci. Remote Sens. 2020, 58, 1665-1677. [CrossRef]

14. Graziano, M.D.; D’Errico, M.; Rufino, G. Wake component detection in X-band SAR images for ship heading and velocity estimation. Remote Sens. 2016, 8, 498. [CrossRef]

15. Tings, B.; Velotto, D. Comparison of ship wake detectability on C-band and X-band SAR. Int. J. Remote Sens. 2018, 39, 4451-4468. [CrossRef]

16. Faltinsen, O.M. Hydrodynamics of High-Speed Marine Vehicles; Cambridge University Press: Cambridge, UK, 2005.

17. Li, Y.; Ellingsen, S.E. Ship waves on uniform shear current at finite depth: Wave resistance and critical velocity. J. Fluid Mech. 2016, 791, 539-567. [CrossRef]

18. Li, Y.; Smeltzer, B.K.; Ellingsen, S.E. Transient wave resistance upon a shear current. Eur. J. Mech. B/Fluids 2019, 73, 180-192. [CrossRef]

19. Fang, M.C.; Yang, R.Y.; Shugan, I.V. Kelvin Ship Wake in the Wind Waves Field and on the Finite Sea Depth. J. Mech. 2011, 27, 71-77. [CrossRef]

20. Shugan, I.V.; Lee, K.J.; Sun, A.J. Kelvin wake in the presence of surface waves. Phys. Lett. A 2006, 357, 232-235. [CrossRef]

21. Akylas, T. Unsteady and nonlinear effects near the cusp lines of the Kelvin ship- wave pattern. J. Fluid Mech. 1987, 175, 333-342. [CrossRef]

22. Chen, H.; Zou, Q. Effects of following and opposing vertical current shear on nonlinear wave interactions. Appl. Ocean Res. 2019, 89, 23-35. [CrossRef]

23. Peregrine, D.H. Interaction of water waves and currents. Adv. Appl. Math. Mech. 1976, 16, 9-117.

24. Teles da Silva, A.F.; Peregrine, D.H. Steep, steady surface waves on water of finite depth with constant vorticity. J. Fluid Mech. 1988, 195, 281-302. [CrossRef]

25. Ellingsen, S.E.; Brevik, I. How linear surface waves are affected by a current with constant vorticity. Eur. J. Phys. 2014, $35,025005$. [CrossRef]

26. Nwogu, O.G. Interaction of finite-amplitude waves with vertically sheared current fields. J. Fluid Mech. 2009, 627, 179-213. [CrossRef]

27. Shrira, V.I. Surface waves on shear currents: Solution of the boundary-value problem. J. Fluid Mech. 1993, 252, 565-584. [CrossRef]

28. Ellingsen, S.E. Initial surface disturbance on a shear current: The Cauchy-Poisson problem with a twist. Phys. Fluids 2014, 26, 082104. [CrossRef]

29. Ellingsen, S.E.; Li, Y. Approximate dispersion relations for waves on arbitrary shear flows. J. Geophys. Res. Ocean. 2017, 122, 9889-9905. [CrossRef]

30. Ellingsen, S.E. Ship waves in the presence of uniform vorticity. J. Fluid Mech. 2014, 742, R2. [CrossRef]

31. Phillips, O.M. The Dynamics of the Upper Ocean, 2nd ed.; Cambridge University Press: Cambridge, UK, 1977. 